in reacting on $\mathrm{CBRN}$-incidents. $64,1 \%$ thought they were not able to act correctly in case of chemical contamination. The most important learning tools were books, lectures, seminars and the principle of learning by doing. The reasons for using an e-learning platform were saving time, high quality of the tutorials (77\% thought it important), quicker reach of information, multimedia formats of the taught facts and links to further information. 55, 2\% were slightly unsatisfied with the actual pool of further trainings. The most frequently used sources of information were the internet (78, $8 \%$ use it frequently) and colleagues.

Conclusions: The survey shows that lacks in disaster preparedness in Germany definitively exist but it also reflects that most of the security and rescue forces are motivated to do further trainings and use therefore new technologies. But they require a high quality of teaching and a reasonable use of them. There is a need for using innovative Methods, and user-friendly web-based instruction and information modules to address all security and rescue forces in Germany.

Prehosp Disaster Med 2011;26(Suppl. 1):s32-s33 doi:10.1017/S1049023X11001178

(A117) Outcomes and Quality of Life after Injury J. Turner, J. Nicholl

Scharr, Sheffield, United Kingdom

Background: Most research into the outcomes of injury has focused on mortality rather than the physical, social, and psychological sequelae of non-fatal injuries. The health and longterm outcomes of a cohort of accidentally-injured patients were studied in order to assess the impact on quality of life.

Methods: The cohort of patients was derived from six previous studies spanning 1988-2003. Patients were followed-up with to ascertain if they were still alive, and survivors were sent a follow-up questionnaire in 2006. The questionnaire asked about current problems resulting from past injuries, use of health services, and measures of health related quality of life (the EQ-5D and SF-36 or Nottingham Health Profile (NHP)). A sample of 114 also received detailed face-to-face interviews.

Results: A total of 2,418 patients were followed-up on between 4-15 years post-injury, of whom 311 had died. There were 580 completed follow-up questionnaires, and of these 64\% reported health problems related to past injuries. The mean EQ-5D score at follow-up was 0.132 tariff points below the mean for a normal age-sex matched population, and SF-36 scores were 5-15 points worse than population norms. At all ages, EQ-5D and SF-36 scores were similar to those of the normal population aged 75 . Interviewed patients also reported substantially more disability than the general population. Increasing injury severity was associated with almost all aspects of worse health at follow-up, and severe lower extremity injuries were strongly associated with poorer outcomes.

Conclusions: Injured patients continue to experience significant reductions in health and health-related quality of life for many years after their injury.

Prehosp Disaster Med 2011;26(Suppl. 1):s33

doi:10.1017/S1049023X1100118X
(A118) Evidence-Based Disaster Medicine: What Can We Learn from a "Science" Spread across 900 Journals? E. Smith, ${ }^{1}$ F.M. Burkle, ${ }^{2}$ P. Aitken, ${ }^{3}$ F. Archer ${ }^{4}$

1. Senior Lecturer, Australia

2. Harvard Humanaitarian Initiative, MA, United States of America

3. Anton Breinl Centre for Public Health and Tropical Medicine, Townsville, Australia

4. Department of Community Emergency Health and Paramedic Practice, Australia

Introduction: Disasters and large-scale crises continue to increase in frequency. To mitigate the potential catastrophes that confront humanity in the new millennium, an evidencebased approach to disaster medicine is required urgently. This paper moves towards such an approach by identifying the current evidence-base for disaster medicine.

Methods: Using a search strategy developed by the Cochrane Prehospital and Emergency Health Field, three independent reviewers searched the electronically indexed database MEDLINE (January 2000 - August 2010) to identify peerreviewed literature relevant to disaster medicine. Reviewers screened the titles and abstracts identified by the search strategy and applied predetermined criteria to classify the reported publications for date, source and study type and topic.

Results: A total of 8149 publications were identified. Of these, $8 \%$ focused on mitigation, $22 \%$ on preparedness, $19 \%$ on response and $51 \%$ on recovery. The publications were overwhelmingly anecdotal or descriptive (89\%) while 5\% were quantitative studies and $6 \%$ used qualitative methodologies. Only 66 of these publications were classified as being high level evidence. The publications were published in 928 journals, of which $34 \%$ were mental health related journals and $28 \%$ were public health journals. The journal "Prehospital and Disaster Medicine" had the greatest number of publications (5\%) of all journals publishing on issues within the scope of disaster medicine. The events with the greatest numbers of publications were the 9/11 terrorist attacks, Hurricane Katrina, the Indian Ocean Tsunami, and the conflict in Iraq. Of note, this search highlights the lack of publications reporting on the application of evaluation tools or frameworks. Conclusion: Given that the "science" of disaster medicine is spread across over 900 different journals, keeping on top of the evolving evidence-base of this emerging discipline will continue to be a challenge. Furthermore, the overall low quality of the evidence is an ongoing concern.

Prebosp Disaster Med 2011;26(Suppl. 1):s33 doi:10.1017/S1049023X11001191

(A119) Ethical Issues in the Review and Conduct of Research during Active Conflicts: Reflections from Darfur, West Sudan

\section{G.M.A. Hussein}

Faculty of Medicine, Riyadh, Saudi Arabia

A crisis has been evolving in the region of Darfur following an armed conflict between rebel groups and the assumingly government-supported militia in 2003. It has attracted international attention and intervention where $13 \mathrm{UN}$ agencies and around 100 national and international non-governmental organizations have been serving the affected populations. Research 